\title{
ApplicationsofElectro-kinetic Principles for Enhancing Durability of Concrete
}

\author{
Mithra $\mathrm{R}^{1}$, R Selvaraj ${ }^{2}$, Om Suganya $^{3}$ \\ ${ }^{I}$ M.Tech,Structural Engineering, VIT University, Vellore, Tamil Nadu, India \\ ${ }^{2}$ Principal Scientist, Corrosion and Material ProtectionDivision, CSIR-CECRI,Karaikudi, Tamil Nadu, India \\ ${ }^{3}$ Assistant Professor, School of Civil and Chemical Engineering, VIT University, Vellore, Tamil Nadu, India
}

\begin{abstract}
Electro-kinetic treatment has been recognized as a promising technology for different purposes in both civil and environmental engineering. The main focus of this study is to improve the durability of cement concrete by application of electro-kinetic method.This method can be used mainly in pre-cast, pre-stressed and reinforced concrete members like railway sleepers, girders and other precast and prefabricated structural elements. Durability of structures is of global concern, many engineers and researchers are working to enhance durability of concrete elements. The durability of concrete can be improved by pore filling effect by ionic migration by adopting electro-kinetic concepts.
\end{abstract}

Keywords:Electro-kinetic treatment, Durability, Pore filling effect, Ionic migration.

\section{Introduction}

Electro-kinetic treatment has been recognized as a promising technology for various purposes in both civil and environmental engineering. The technique utilizes the application of an electric DC field for transportation of ions(electro-migration) [1-3].In the field of civil engineering, particularly for soil remediation, chlorides, sulphates and anaerobic bacteria are removed by applying the principles of electro-kinetics[4-7]. Also, as per recent studies in decades the application of electro-kinetic principles is useful for desalination of bricks, natural stones and tiles[8]. It can be stated that the electro-kinetic principles are applied for recovering heavy metals like Cadmium, Lead, Chromium and Zinc etc...

Applying the principle of electro-kinetics for enhancing durability of concrete, that is electro-migration of ions from the selected electrolytes with selected applied DC voltage. Pores, voids, fissures, cracks and other surface defects of prefabricated elements can be filled thoroughly by this ionic migration. Usually the pore fillers are of cations such as Calcium, Magnesium, Aluminium and Titanium.

In this paper, it is suggested that an electro-kinetic principle with selected electrolyte, applied voltage with appropriate electrode materials can be utilized for enhancing the durability by filling the pores andsurface defects of concrete.

\section{General Principles of Electro-kinetics}

Electro-kinetic transport is the phenomenon of charged particles moving in response to an applied electric field. Electro-kinetic transport includes ionic conduction, electrophoresis, and electro-osmosis. Ionic solution conductivity accounts for the overwhelming majority of conductivity measured in cement based materials. In an aqueous system (cement concrete structures generally retain certain moisture content in most conditions), ions can be induced to drift in response to an applied electronic field.Electro-osmosis is the induced flow of water through a porous medium such as sand, clay or concrete when an electric potential is applied across the medium. Electrophoresis is characterized by the movement of a solid particle dispersed in an electrolyte under the influence of an electric field. [1-3].

\section{Electro-kinetics Principles applied to soil remediation:}

Electro-kinetic method of soil remediation technique involves application of low voltage direct current (DC) in the order of 1 volt per $\mathrm{cm}$ distance between anode and cathode or current is applied in the order of $\mathrm{mA} / \mathrm{cm}^{2}$ of the electrode area. The soil is the medium and water acts as electrolyte. This low level DC results inhydrological and physio-chemical changes in the soil mass resulting in species transport in the porous soilmedium, voids, water, organic, decomposed organic matters, mild organic acids and microbes [11] 


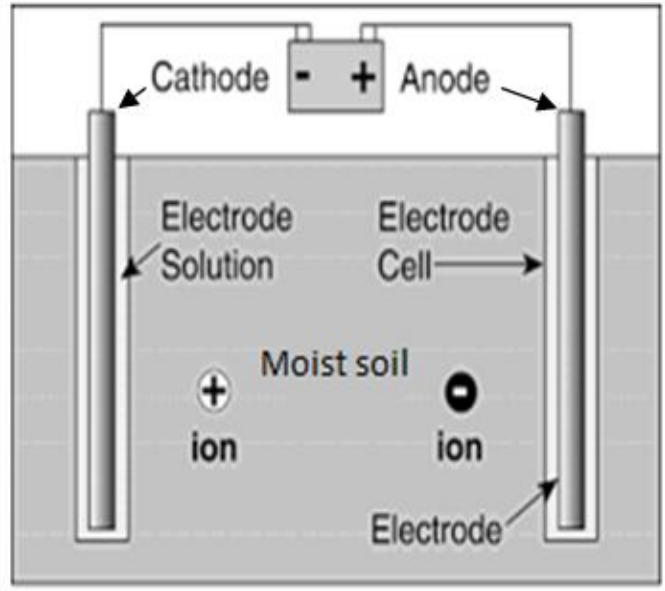

Fig 1:Principle of electro-kinetics for soil remediation

Electro-kinetic setup for Durability enhancement of concrete:

The test mortar of 1:3 hasbeen chosen for the study.

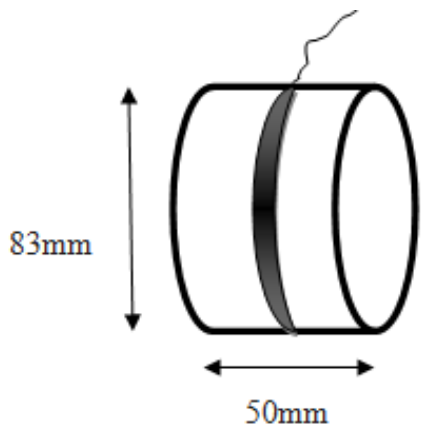

Fig2: Test Specimen

Specimens of size $83 \mathrm{~mm}$ diameter and $50 \mathrm{~mm}$ depth are chosen. A wire mesh of stainless steel is inserted at $25 \mathrm{~mm}$ depth and a lead is taken out to act as cathode. An electrode is placed to act as anode. They are immersed in a container with electrolyte solution which can beMagnesium hydroxide $\left(\mathrm{Mg}(\mathrm{OH})_{2}\right)$, Calcium carbonate $\left(\mathrm{CaCO}_{3}\right)$, Titanium oxide $\left(\mathrm{TiO}_{2}\right)$ or Aluminum oxide $\left(\mathrm{Al}_{2} \mathrm{O}_{3}\right)$ with varying concentrations. For our study, potential can be variedwith varying duration of potential.

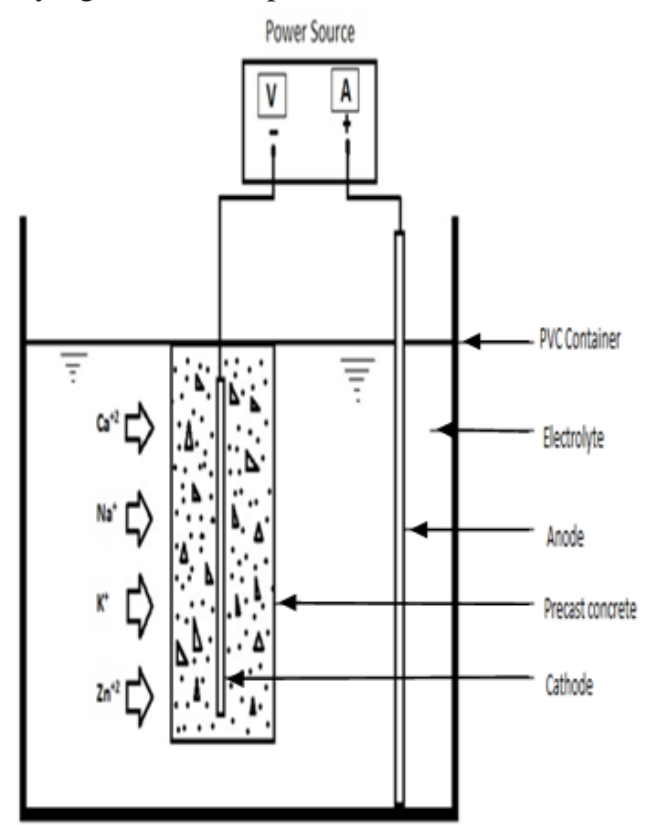

Fig 3:Electro-Kinetic treatment setup 
Electro-kinetics is based on the fundamentals of electrolysis, where charged species are transported towards the oppositely charged electrodes under the action of an applied electric field[1-3]. The primary electrode reactions are

(1)Oxidation of water at the anode generating acid.

(2)Reduction of water at the cathode,producing an alkaline front.

$$
\longrightarrow \quad \mathrm{H}_{2} \mathrm{O} \quad 2 \mathrm{H}^{+}+1 / 2 \mathrm{O}_{2}(\mathrm{~g})+2 \mathrm{e}^{-}(1)
$$

However in liquid electrolyte, this effect of electrolysis is nullified.Concrete is a mixture of sand, stone (or aggregates) together with a hardened cement paste that is porous. The dominant micro-structural feature governing transport is the pore structure of concrete. Pore structure originates from the microstructure when water, anhydrous cement grains, and aggregates are mixed. Capillary pore structure initially assumes the shape of the space occupied by mix water. However, hydration of the cement yields calcium silicate hydrate $(\mathrm{C}-\mathrm{S}-$ $\mathrm{H}$ ) the primary binder in hardened cement paste. As these hydration products formthe capillary pore structure is developed, capillary pores tend to dominate transport processes. Pore volume of the sample, size distribution, tortuosity, and connectivity are the specific structural characteristics of capillary pores which influence transport. Micro-cracks and bleed paths also influence particle transport. Micro-cracks form during drying of the calcium silicate hydrate layers which shrink and lead to tensile stress and cracking. Tensile stresses lead to plastic shrinkage, stresses from applied loads, thermal expansion or freezing pore water may also induce microcracking. Bleed paths occur prior to setting, water accumulates around aggregates and moves toward the surface of the cement paste. Discrete flows can join together to form bleed paths which remain after setting of the cement paste.

Changes in water content of hardened cement pastes have significant impacts on transport mechanisms and rates. At relative humidity above $45 \%$, evaporable pore water is said to exist. Above this threshold, while the permeability of gases is increasingly blocked by liquid water barriers, the transport of aqueous ions or particles progresses more rapidly as the presence of evaporable capillary water increases[10].Proposed evaluation tests for enhancing durability of concrete after electro-kinetic process are: Chloride Ion Penetration test (ASTM C 1202), Impressed voltage test,Water absorption and Sorptivity test.

\section{References}

[1]. GrySkibsted, Lisbeth M Ottosen and PernilleE.Jensen(2013),Electro-chemical desalination of bricks-removal of salts.

[2]. GrySkibsted, Lisbeth M Ottosen and PernilleE.Jensen(2010), The possibility of using electro-kinetics of desalinatiom of sandstone with low porosity.

[3]. GrySkibsted, Lisbeth M Ottosen and PernilleE.Jensen(2013), Long term electrodialytic soil remediation-effect of removal efficiency of lead.

[4]. BertoliniL,Yu SW, Page CL(1996).Effects of electrochemical chloride extraction on chemical and mechanical properties of hydrated cement paste, Adv Cement Res8(31):93-100

[5]. Mietz.(1998).Electrochemical rehabilitation methods for reinforced concrete structures. Publication no.24 of the European Federation of Corrosion, The Institute of Materials, London

[6]. Hosseini A, KhalooAR(2005).Study of electrochemical chloride extraction as a non-destructive repair method:Part 1.Discrete test samples. Asian J CivEng (Buildings and Housing) 6(3),167-182

[7]. Fajardo G, Escadeillas G, Argiguie G(2006).Electrochemical chloride extraction (ECE) from steel-reinforced concrete specimens contaminated by "artificial" sea-water, CorrosSci 48:110-125

[8]. Ottosen LM, Rorig-DalgaardI(2009), Desalination of a brick by application of an electric DC field. Materials and Structures 42:961-971

[9]. Lagemen R, Pool W, Seffinga G(1989), Electro-Reclamation- theory and practice, chemistry and industry 18:585-590

[10]. US 8377278 B1 Electro-kinetic strength enhancement of concrete

[11]. M Amirthavarshini, R Selvaraj(2016), Rudiments of Electro-kinetic method of soil remediation in civil engineering. 